\title{
Antimicrobial Activity of Some Derivatives of 1,4-Dihydropyridines
}

\author{
Prabha Mehta and Prabha Verma \\ Department of Chemistry, Government Autonomous K.R.G. College, Gwalior 474002, India \\ Correspondence should be addressed to Prabha Mehta; prabhamehta6@gmail.com
}

Received 7 December 2011; Accepted 30 August 2012

Academic Editor: Franck Le Derf

Copyright (c) 2013 P. Mehta and P. Verma. This is an open access article distributed under the Creative Commons Attribution License, which permits unrestricted use, distribution, and reproduction in any medium, provided the original work is properly cited.

Hantzsch reported the synthesis of functionalized 1,4- dihydropyridines via three-component condensation of an aromatic aldehyde, ketoester, and ammonium hydroxide. This multicomponent reaction is of much importance due to excellent pharmacological properties of dihydropyridines. In this account, we synthesized some halo- and nitrophenyl dihydropyridines and evaluated their antimicrobial activity. The minimum inhibitory concentration (MIC) was determined by microdilution technique in Mueller Hinton broth. The MICs were recorded after 24 hours of incubation at $37^{\circ} \mathrm{C}$. These results showed that these compounds exhibited significant to moderate activities against both Gram-(+) and Gram-(-) organisms.

\section{Introduction}

Antimicrobial drugs are the greatest contributions in the present century to therapeutics. It is essential to investigate newer drugs with lesser resistances. Systematic studies among various pharmaceutical compounds have revealed that any drug may possess diverse functions. Drugs belonging to different pharmacological classes such as vasodilators, antihypertensive [1-4], antiinflammatory [5], anaesthetics [6], anti-ischemic [7], and calcium channel modulators of the nifedipine type [8] may have useful activity in completely different spheres of medicine. Amlodipine and lacidipine, dihydropyridine $\mathrm{Ca}^{++}$channels blockers, are used orally for the treatment of hypertension. Previous papers suggested that amlodipine can also inhibit the proliferation of different cancer cells $[9,10]$. It has also been reported that lacidipine [11] and some 3-chlorophenyl [12], nitrophenyl 1,4-dihydropyridine [13] derivatives are cytotoxic towards trypanosoma cruzi through respiratory chain inhibition. Amlodipine and lacidipine both contain a phenyl-1,4dihydropyridine ring which is absent in verapamil and diltiazem. In view of these observations, it was decided to synthesize a new series of 1,4-dihydropyridine derivatives ( $\mathrm{V}-1$ to $\mathrm{V}-9$ ) and screen them for their level of antimicrobial activity.

\section{Experimental}

2.1. General Procedure for Synthesis of Substituted 1,4Dihydropyridines ( $V-1$ to $V-9)$. The mixture of aryl aldehyde (1 mole), $\beta$-ketoester ( 2 mole), and ammonium hydroxide $(8 \mathrm{~mL})$ was refluxed together in ethanol for about 5-18 hours (Scheme 1). Then the reaction mixture was cooled and recrystallized with ethyl alcohol.

Synthesis of 3,5-Diethyl-2,6-dimethy-4-(3-nitrophenyl)-1,4-dihydropyridine-3,5-dicarboxylate $(V-1)$. The reaction mixture consisting of $\mathrm{m}$-nitrobenzaldehyde $(0.1 \mathrm{~mol})$, ethyl acetoacetate $(0.2 \mathrm{~mol})$, and ammonium hydroxide $(8 \mathrm{~mL})$ in ethanol $(60 \mathrm{~mL})$ was heated at reflux for 5 hours. The obtained solid was filtered off, washed with warm water and recrystallized using ethyl alcohol. The above procedure was followed for the synthesis of compounds V-2 and V-5.

Synthesis of 3-Ethyl-5-(1,1-dimethyl ethyl)-2,6-dimethyl-4-(3chlorophenyl)-1,4-dihydropyridine-3,5-dicarboxylate (V-6). The reaction mixture consisting of chlorobenzaldehyde $(0.025 \mathrm{~mol})$, ethyl acetoacetate $(0.025 \mathrm{~mol}), \mathrm{t}$-butyl acetoacetate $(0.025 \mathrm{~mol})$, and ammonium hydroxide $(2 \mathrm{~mL})$ in ethanol $(15 \mathrm{~mL})$ was heated at reflux for 11 hours. 
<smiles>[R]C(=O)CC([R6])[R]</smiles>

Scheme 1: Synthesis of 1,4-dihydropyridines.

The obtained solid was filtered off, washed with warm water, and recrystallized using ethyl alcohol. The above procedure was followed for the synthesis of compounds V-8 and V-9.

Synthesis of 3,5-Di(1,1-dimethyl ethyl)-2,6-dimethyl-4-(3chlorophenyl)-1,4-dihydropyridine-3,5-dicarboxylate (V-3). The reaction mixture consisting of $\mathrm{m}$-chlorobenzaldehyde $(0.02 \mathrm{~mol})$, t-butyl acetoacetate $(0.04 \mathrm{~mol})$, and ammonium hydroxide $(1.6 \mathrm{~mL})$ in ethanol $(12 \mathrm{~mL})$ was heated at reflux for 8 hours. The obtained solid was filtered off, washed with warm water, and recrystallized using ethyl alcohol. The above procedure was followed for the synthesis of compound V-7 (see Table 1).

Synthesis of 3,5-Di(1,1-dimethyl ethyl)-2,6-dimethyl-4-(3-nitrophenyl)-1,4-dihydropyridine-3,5-dicarboxylate $V$-4). The reaction mixture consisting of $\mathrm{m}$-nitrobenzaldehyde ( 0 . $016 \mathrm{~mol})$, t-butyl acetoacetate $(0.032 \mathrm{~mol})$, and ammonium hydroxide $(1.33 \mathrm{~mL})$ in ethanol $(10 \mathrm{~mL})$ was heated at reflux for 10 hours. The obtained solid was filtered off, washed with warm water, and recrystallized using ethyl alcohol.

2.2. Spectral Data of Compound (V-1 to V-9). 3,5-Diethyl2,6-dimethy-4-(3-nitrophenyl)-1,4-dihydropyridine-3,5-dicarboxylate, $V-1$. IR $\left(\mathrm{KBr} \mathrm{cm}^{-1}\right)$ : 3344 (-NH str), 2988(C-H), 1705 (C=O, ester), $1526\left(-\mathrm{NO}_{2}\right) ;{ }^{1} \mathrm{HNMR}\left(400 \mathrm{MHz}, \mathrm{CDCl}_{3}\right)$ : 8.12(s, 1H, NH), 7.98-8.01 (m, $4 \mathrm{H}$ Ph-ring), 5.09 (s, $1 \mathrm{H}$, $\mathrm{CH}), 4.02-4.15\left(\mathrm{~m}, 4 \mathrm{H}, 2 \mathrm{CH}_{2}\right.$, ester group), $2.36(\mathrm{~s}, 6 \mathrm{H}$, $2 \mathrm{CH}_{3}$ ester group), $1.20-1.23\left(\mathrm{t}, 6 \mathrm{H}, 2 \mathrm{CH}_{3}\right.$, methyl group); $m / z: 252,(M=100 \%)$.

3,5-Diethyl-2,6-dimethy-4-(3-chlorophenyl)-1,4-dihydropyridine-3,5-dicarboxylate, $V-2$. IR $\left(\mathrm{KBr} \mathrm{cm}^{-1}\right)$ : $3322(-\mathrm{NH}$ str), 2980(C-H), 1703 (C=O, ester), 620(-Cl); ${ }^{1} \mathrm{HNMR}$ $\left(400 \mathrm{MHz}, \mathrm{CDCl}_{3}\right): 7.26(\mathrm{~s}, 1 \mathrm{H}, \mathrm{NH}), 7.08-7.18(\mathrm{~m}, 4 \mathrm{H}$, Ph-ring), $4.96(\mathrm{~s}, 1 \mathrm{H}, \mathrm{CH}), 4.04-4.14\left(\mathrm{~m}, 4 \mathrm{H}, 2 \mathrm{CH}_{2}\right.$ ester group), 2.33 (s, $6 \mathrm{H}, 2 \mathrm{CH}_{3}$, methyl group), $1.20-1.24(\mathrm{t}, 6 \mathrm{H}$, $2 \mathrm{CH}_{3}$, ester group); $m / z: 252,(M=100 \%)$.

3,5-Di(1,1-dimethylethyl)-2,6-dimethyl-4-(3-chlorophenyl)1,4-dihydropyridine-3,5-dicarboxylate, $V$-3. IR $\left(\mathrm{KBr} \mathrm{cm}^{-1}\right)$ : 3334(-NH str), 2973(C-H), 1698 (C=O, ester), 612(-Cl); ${ }^{1} \mathrm{HNMR}\left(400 \mathrm{MHz}, \mathrm{CDCl}_{3}\right): 7.26(\mathrm{~s}, 1 \mathrm{H}, \mathrm{NH}), 7.07-7.17$ (m, $4 \mathrm{H}$, Ph-ring), 4.88 (s, 1H, CH), 2.29 (s, 6H, 2CH 3 , methyl group), $1.40\left(\mathrm{~s}, 18 \mathrm{H}, 2\left(\mathrm{CH}_{3}\right)_{3}\right) ; m / z: 152,(M=100 \%)$.

3,5-Di(1,1-dimethylethyl)-2,6-dimethyl-4-(3-nitrophenyl)-

1,4-dihydropyridine-3,5-dicarboxylate, $V$-4. IR $\left(\mathrm{KBr} \mathrm{cm}^{-1}\right)$ : 3337 (-NH str), $2972(\mathrm{C}-\mathrm{H}), 1699(\mathrm{C}=\mathrm{O}$, ester), 1528 $\left(-\mathrm{NO}_{2}\right) ;{ }^{1} \mathrm{HNMR}\left(400 \mathrm{MHz}, \mathrm{CDCl}_{3}\right): 8.16(\mathrm{~s}, 1 \mathrm{H}, \mathrm{NH})$, 7.99-8.01 (m, 4H, Ph-ring), 5.01(s, 1H, CH), 2.31 (s, 6H, $2 \mathrm{CH}_{3}$, methyl group), $1.39\left(\mathrm{~s}, 18 \mathrm{H}, 2\left(\mathrm{CH}_{3}\right)_{3}\right.$, ester group); $m / z: 108,(M=100 \%)$.

3,5-Diethyl-2,6-dimethy-4-(2-chlorophenyl)-1,4-dihydropyridine-3,5-dicarboxylate, $V-5$. IR $\left(\mathrm{KBr} \mathrm{cm}^{-1}\right)$ : $3332(-\mathrm{NH}$ str), 2978(C-H), 1659 (C=O, ester), 623(-Cl); ${ }^{1} \mathrm{HNMR}(400$ $\mathrm{MHz}, \mathrm{CDCl}_{3}$ ): 7.26 (s, 1H, NH), 7.09-7.23 (m, 4H, Ph-ring), $5.39(\mathrm{~s}, 1 \mathrm{H}, \mathrm{CH}), 4.04-4.10\left(\mathrm{~m}, 4 \mathrm{H}, 2 \mathrm{CH}_{2}\right.$ ester group), $2.27\left(\mathrm{~s}, 6 \mathrm{H}, 2 \mathrm{CH}_{3}\right.$, ester group); $m / z: 252,(M=100 \%)$.

3-Ethyl-5-(1,1-dimethyl ethyl)-2,6-dimethyl-4-(3-chloro phenyl)-1,4-dihydropyridine-3,5-dicarboxylate, $V$-6. IR $\left(\mathrm{KBr} \mathrm{cm}^{-1}\right)$ : 3312 (-NH str), 2981(C-H), 1697 (C=O, ester), $682(-\mathrm{Cl}) ;{ }^{1} \mathrm{HNMR}\left(400 \mathrm{MHz}, \mathrm{CDCl}_{3}\right): 7.26$ (s, $\left.1 \mathrm{H}, \mathrm{NH}\right)$, 7.15-7.18 (m, 4H, Ph-ring), 4.96 (s, 1H, CH), 4.04-4.12 (m, $2 \mathrm{H}, \mathrm{CH}_{2}$, ester group), 2.33 (s, $6 \mathrm{H}, 2 \mathrm{CH}_{3}$, methyl group), $1.39\left(\mathrm{~s}, 9 \mathrm{H},\left(\mathrm{CH}_{3}\right)_{3}\right.$, ester group) $1.22-1.24\left(\mathrm{t}, 3 \mathrm{H}, \mathrm{CH}_{3}\right.$, ester group); $m / z: 224,(M=100 \%)$.

3,5-Di(1,1-dimethyl ethyl)-2,6-dimethyl-4-(2-chlorophenyl)-1, 4-dihydropyridine-3,5-dicarboxylate, $V-7$. IR $\left(\mathrm{KBr} \mathrm{cm}^{-1}\right)$ : 3341 (-NH str), $2976(\mathrm{C}-\mathrm{H}), 1699$ (C=O, ester), $645(-\mathrm{Cl})$; ${ }^{1} \mathrm{HNMR}\left(400 \mathrm{MHz}, \mathrm{CDCl}_{3}\right): 7.38$ (s, 1H, NH), 7.22-7.26 (m, $4 \mathrm{H}$, Ph-ring), 5.23 (s, $1 \mathrm{H}, \mathrm{CH}), 2.22\left(\mathrm{~s}, 6 \mathrm{H}, 2 \mathrm{CH}_{3}\right.$, methyl group), 1.37 (s, $18 \mathrm{H}, 2\left(\mathrm{CH}_{3}\right)_{3}$, ester group).

3-Ethyl-5-(1,1-dimethyl ethyl)-2,6-dimethyl-4-(2-chloro phenyl)-1,4-dihydropyridine-3,5-dicarboxylate, $\quad V-8$. IR $\left(\mathrm{KBr} \mathrm{cm}^{-1}\right)$ : 3327(-NH str), 2981(C-H), 1678 (C=O, ester), 639 (-Cl); ${ }^{1} \mathrm{HNMR}\left(400 \mathrm{MHz}, \mathrm{CDCl}_{3}\right): 7.39$ (s, 1H, NH), 7.22-7.26 (m, 4H, Ph-ring), 4.76 (s, $1 \mathrm{H}, \mathrm{CH}), 4.02-4.10$ (m, $2 \mathrm{H}, \mathrm{CH}_{2}$, ester group), $2.27\left(\mathrm{~s}, 6 \mathrm{H}, 2 \mathrm{CH}_{3}\right.$, methyl group), $1.38\left(\mathrm{~s}, 9 \mathrm{H},\left(\mathrm{CH}_{3}\right)_{3}\right.$, ester group), $1.19-1.22\left(\mathrm{t}, 3 \mathrm{H}, \mathrm{CH}_{3}\right.$, ester group); $m / z: 252,(M=100 \%)$.

3,5-Di(1,1-dimethyl ethyl)-2,6-dimethyl-4-(2-nitrophenyl)-1,4 -dihydropyridine-3,5-dicarboxylate, $V$-9. IR $\left(\mathrm{KBr} \mathrm{cm}^{-1}\right): 3342$ (-NH str), $2975(\mathrm{C}-\mathrm{H}), 1693\left(\mathrm{C}=\mathrm{O}\right.$, ester), $1531\left(-\mathrm{NO}_{2}\right)$; ${ }^{1} \mathrm{HNMR}\left(400 \mathrm{MHz}, \mathrm{CDCl}_{3}\right.$ ): 7.52 (s, 1H, NH), 7.22-7.47 (m, $4 \mathrm{H}$, Ph-ring), 5.44 (s, $1 \mathrm{H}, \mathrm{CH}), 2.27$ (s, 6H, 2CH 3 , methyl group), 1.36 (s, 18H, $2\left(\mathrm{CH}_{3}\right)_{3}$, ester group). 
TABLE 1: Analytical data of compounds V-1 to V-9.

\begin{tabular}{|c|c|c|c|c|c|c|c|c|c|}
\hline \multirow{2}{*}{ Compound } & \multirow{2}{*}{ Mol weight } & \multirow{2}{*}{ Molecular formula } & \multirow{2}{*}{ M.P $\left({ }^{\circ} \mathrm{C}\right)$} & \multirow{2}{*}{ Tim (hr) } & \multirow{2}{*}{ Yield (\%) } & \multicolumn{4}{|c|}{ Found \% (Cald) } \\
\hline & & & & & & $\mathrm{C}$ & $\mathrm{H}$ & $\mathrm{N}$ & $\mathrm{Cl}$ \\
\hline $\mathrm{V}-1$ & 374 & $\mathrm{C}_{19} \mathrm{H}_{22} \mathrm{~N}_{2} \mathrm{O}_{6}$ & 165 & 5 & 48.12 & $\begin{array}{c}60.93 \\
(60.96)\end{array}$ & $\begin{array}{c}5.91 \\
(5.88)\end{array}$ & $\begin{array}{c}7.46 \\
(7.48)\end{array}$ & $\begin{array}{l}- \\
-\end{array}$ \\
\hline $\mathrm{V}-2$ & 363.5 & $\mathrm{C}_{19} \mathrm{H}_{22} \mathrm{NO}_{4} \mathrm{Cl}$ & 120 & 5 & 47.96 & $\begin{array}{c}62.69 \\
(62.72)\end{array}$ & $\begin{array}{c}6.06 \\
(6.05)\end{array}$ & $\begin{array}{c}3.83 \\
(3.85)\end{array}$ & $\begin{array}{c}9.77 \\
(9.76)\end{array}$ \\
\hline $\mathrm{V}-3$ & 419.5 & $\mathrm{C}_{23} \mathrm{H}_{30} \mathrm{NO}_{4} \mathrm{Cl}$ & 176 & 8 & 18.10 & $\begin{array}{c}65.74 \\
(65.79)\end{array}$ & $\begin{array}{c}7.17 \\
(7.15)\end{array}$ & $\begin{array}{c}3.32 \\
(3.33)\end{array}$ & $\begin{array}{c}8.48 \\
(8.46)\end{array}$ \\
\hline $\mathrm{V}-4$ & 430 & $\mathrm{C}_{23} \mathrm{H}_{30} \mathrm{~N}_{2} \mathrm{O}_{6}$ & 188 & 10 & 10.18 & $\begin{array}{c}64.15 \\
(64.18)\end{array}$ & $\begin{array}{c}6.98 \\
(6.97)\end{array}$ & $\begin{array}{c}6.50 \\
(6.51)\end{array}$ & - \\
\hline V-5 & 363.5 & $\mathrm{C}_{19} \mathrm{H}_{22} \mathrm{NO}_{4} \mathrm{Cl}$ & 132 & 16 & 41.69 & $\begin{array}{c}62.69 \\
(62.72)\end{array}$ & $\begin{array}{c}6.04 \\
(6.02)\end{array}$ & $\begin{array}{c}3.82 \\
(3.85)\end{array}$ & $\begin{array}{c}9.74 \\
(9.76)\end{array}$ \\
\hline V-6 & 391.5 & $\mathrm{C}_{21} \mathrm{H}_{26} \mathrm{NO}_{4} \mathrm{Cl}$ & 108 & 11 & 20.22 & $\begin{array}{c}64.39 \\
(64.36)\end{array}$ & $\begin{array}{c}6.62 \\
(6.64)\end{array}$ & $\begin{array}{c}3.72 \\
(3.75)\end{array}$ & $\begin{array}{c}9.05 \\
(9.06)\end{array}$ \\
\hline $\mathrm{V}-7$ & 419.5 & $\mathrm{C}_{23} \mathrm{H}_{30} \mathrm{NO}_{4} \mathrm{Cl}$ & 168 & 13 & 11.55 & $\begin{array}{c}65.70 \\
(65.79)\end{array}$ & $\begin{array}{c}7.14 \\
(7.15)\end{array}$ & $\begin{array}{c}3.35 \\
(3.33)\end{array}$ & $\begin{array}{c}8.43 \\
(8.46)\end{array}$ \\
\hline $\mathrm{V}-8$ & 391.5 & $\mathrm{C}_{21} \mathrm{H}_{26} \mathrm{NO}_{4} \mathrm{Cl}$ & 112 & 13 & 12.30 & $\begin{array}{c}64.39 \\
(64.36)\end{array}$ & $\begin{array}{c}6.62 \\
(6.64)\end{array}$ & $\begin{array}{c}3.74 \\
(3.75)\end{array}$ & $\begin{array}{c}9.03 \\
(9.06)\end{array}$ \\
\hline V-9 & 430 & $\mathrm{C}_{23} \mathrm{H}_{30} \mathrm{~N}_{2} \mathrm{O}_{6}$ & 109 & 15 & 11.81 & $\begin{array}{c}64.16 \\
(64.18)\end{array}$ & $\begin{array}{c}6.99 \\
(6.97)\end{array}$ & $\begin{array}{c}6.50 \\
(6.51)\end{array}$ & $\begin{array}{l}- \\
-\end{array}$ \\
\hline
\end{tabular}

TABle 2: Antibacterial activity of compounds V-1 to V-9 against Gram-(+) and Gram-(-) organisms.

\begin{tabular}{lccccc}
\hline S. no. & $\begin{array}{c}\text { Compounds } \\
\text { name }\end{array}$ & E. coli & $\begin{array}{c}\text { Km }(-) \\
\text { aerogenes }\end{array}$ & NLF & S. aureus \\
\hline$(1)$ & $\mathrm{V}-1$ & + & $\mathrm{R}$ & + & $\mathrm{R}$ \\
$(2)$ & $\mathrm{V}-2$ & ++ & $\mathrm{R}$ & +++ & $\mathrm{R}$ \\
$(3)$ & $\mathrm{V}-3$ & $\mathrm{R}$ & +++ & + & +++ \\
$(4)$ & $\mathrm{V}-4$ & ++ & ++ & $\mathrm{R}$ & $\mathrm{R}$ \\
$(5)$ & $\mathrm{V}-5$ & $\mathrm{R}$ & $\mathrm{R}$ & $\mathrm{R}$ & + \\
$(6)$ & $\mathrm{V}-6$ & $\mathrm{R}$ & $\mathrm{R}$ & $\mathrm{R}$ & + \\
$(7)$ & $\mathrm{V}-7$ & + & $\mathrm{R}$ & $\mathrm{R}$ & ++ \\
$(8)$ & $\mathrm{V}-8$ & $\mathrm{R}$ & $\mathrm{R}$ & $\mathrm{R}$ & $\mathrm{R}$ \\
$(9)$ & $\mathrm{V}-9$ & $\mathrm{R}$ & $\mathrm{R}$ & $\mathrm{R}$ & $\mathrm{R}$ \\
\hline
\end{tabular}

Zone of inhibition was measured in mm. +++: strong activity, ++: good activity, +: moderate activity, R: resistant.

2.3. Antimicrobial Studies. In vitro antibacterial studies of the newly synthesized compounds V-1 to V-9 were screened for their in vitro antibacterial activity against Escherichia coli, Klebsiella aerogenes, nonlactose fermenters, and Staphylococcus aureus according to the disc diffusion method. The minimum inhibitory concentration (MIC) was determined by the serial dilution technique using acetone as solvent. The zone of inhibition is reported in the Table 2. Nutrient agar was employed as culture medium. The MIC calculated was $400 \mu$ $\mathrm{g} / \mathrm{mL}$ for compounds.

\section{Results and Discussion}

The synthesis of a series of 1,4-dihydropyridine derivatives $(\mathrm{V}-1)$ to $(\mathrm{V}-9)$ has been achieved by condensation of acetoacetic ester, aryl aldehyde, and ammonium hydroxide in ethanol. All the synthesized 1,4 dihydropyridines have given appreciable yield with satisfactory element analysis. The compounds V-1 to V-9 were evaluated for their in vitro antibacterial activity against Gram-(-) and Gram-(+) organisms. It is inferred from Table 2, out of nine synthesized compounds V-1 to V-9, compound V-2 was effective against Gram-(-) organism, that is, E. coli and NLF. Compound V3 was effective against Gram-(-) and Gram-(+) organisms, that is, against Klebsiella aerogenes and Staphylococcus aureus, while compounds $\mathrm{V}-1$ and V-4 were only effective against Gram-(-) E. coli and NLF.

\section{Acknowledgments}

The authors wish to express their thanks and gratitude to the Principal Government Autonomous K.R.G. College, Gwalior for providing necessary facilities. They sincerely thank Dr. S. C. Mehta, Professor in the Department of Pharmacology and Dr. (Mrs.) Shashi Gandhi, Professor in the Department of Microbiology, G.R. Medical College, Gwalior for their help and carrying out biological activities. This paper was supported by UGC grant.

\section{References}

[1] A. C. Gaudio, A. Korolkovas, and Y. Takahata, "Quantitative structure-activity relationships for 1,4-dihydropyridine calcium channel antagonists (nifedipine analogues): a quantum chemical/classical approach," Journal of Pharmaceutical Sciences, vol. 83, no. 8, pp. 1110-1115, 1994.

[2] K. J. Chelifer, "Stereoselective characterization of the 1,4dihydropyridine binding site at L-type calcium channels in 
the resting state and the opened/inactivated state," Journal of Medicinal Chemistry, vol. 42, no. 12, pp. 2204-2211, 1999.

[3] S. G. Dastidar, U. Mondal, S. Niyogi, and A. N. Chakrabarty, "Antibacterial property of methyl-DOPA and development of antibiotic cross-resistances in m-DOPA mutants," Indian Journal of Medical Research, vol. 84, no. 2, pp. 142-147, 1986.

[4] K. A. Kumar, K. Ganguly, K. Mazumdar, N. K. Dutta, S. G. Dastidar, and A. N. Chakrabarty, "Amlodipine: a cardiovascular drug with powerful antimicrobial property," Acta Microbiologica Polonica, vol. 52, no. 3, pp. 285-292, 2003.

[5] S. Annadurai, S. Basu, S. Ray, S. G. Dastidar, and A. N. Chakrabarty, "Antibacterial activity of the antiinflammatory agent diclofenac sodium," Indian Journal of Experimental Biology, vol. 36, no. 1, pp. 86-90, 1998.

[6] S. G. Dastidar, S. Das, M. Mookerjee, D. Chattopadhyay, S. Ray, and A. N. Chakrabarty, "Antibacterial activity of local anaesthetics procaine and lignocaine," Indian Journal of Medical Research, vol. 87, no. 5, pp. 506-508, 1988.

[7] B. Khadilkar and S. Borkar, "Silica gel supported ferric nitrate: a convenient oxidizing reagent," Synthetic Communications, vol. 28, no. 2, pp. 207-212, 1998.

[8] B. Schnell, W. Krenn, K. Faber, and C. O. Kappe, "Synthesis and reactions of Biginelli-compounds. Part 23. Chemoenzymatic syntheses of enantiomerically pure 4-aryl3,4-dihydropyrimidin-2(1H)-ones," Journal of the Chemical Society, Perkin Transactions, vol. 24, pp. 4382-4389, 2000.

[9] X. Li, G. R. Ruan, W. L. Lu et al., "A novel stealth liposomal topotecan with amlodipine: apoptotic effect is associated with deletion of intracellular $\mathrm{Ca}^{2+}$ by amlodipine thus leading to an enhanced antitumor activity in leukemia," Journal of Controlled Release, vol. 112, no. 2, pp. 186-198, 2006.

[10] J. M. Taylor and R. U. Simpson, "Inhibition of cancer cell growth by calcium channel antagonists in the athymic mouse," Cancer Research, vol. 52, no. 9, pp. 2413-2418, 1992.

[11] L. J. Núñez-Vergara, J. A. Squella, S. Bollo-Dragnic et al., "Isradipine and lacidipine: effects in vivo and in vitro on Trypanosoma cruzi epimastigotes," General Pharmacology, vol. 30, no. 1, pp. 85-87, 1998.

[12] J. D. Maya, A. Morello, Y. Repetto et al., "Effects of 3-chlorophenyl-1,4-dihydropyridine derivatives on Trypanosome cruzi epimastigotes," Comparative Biochemistry and Physiology, vol. 125, pp. 103-109, 2000.

[13] L. J. Núñez-Vergara, J. A. Squella, S. Bollo-Dragnic et al., "Nitro aryl 1,4-dihydropyridine derivatives: effects of Trypanosoma cruzi," Comparative Biochemistry and Physiology, vol. 118, no. 1, pp. 105-111, 1997. 

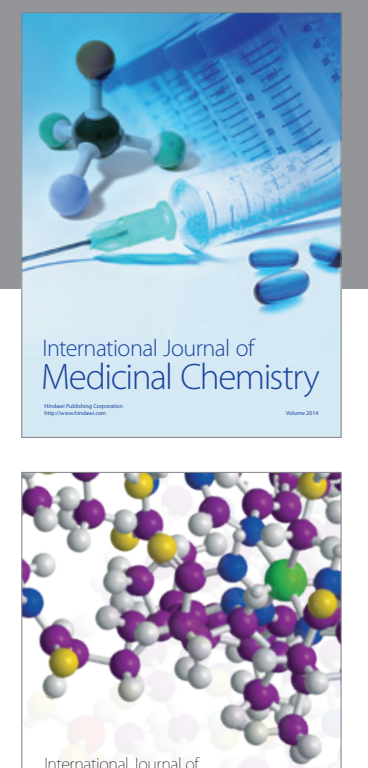

\section{Carbohydrate} Chemistry

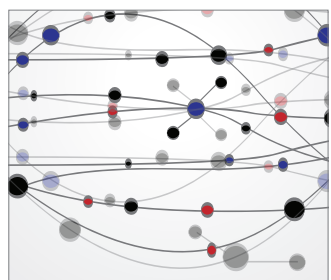

The Scientific World Journal
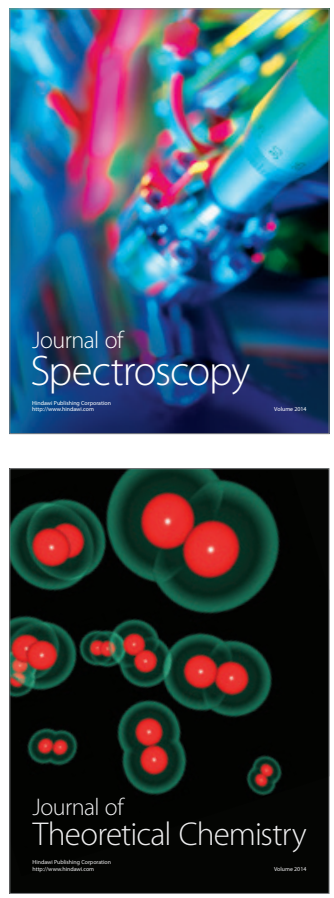
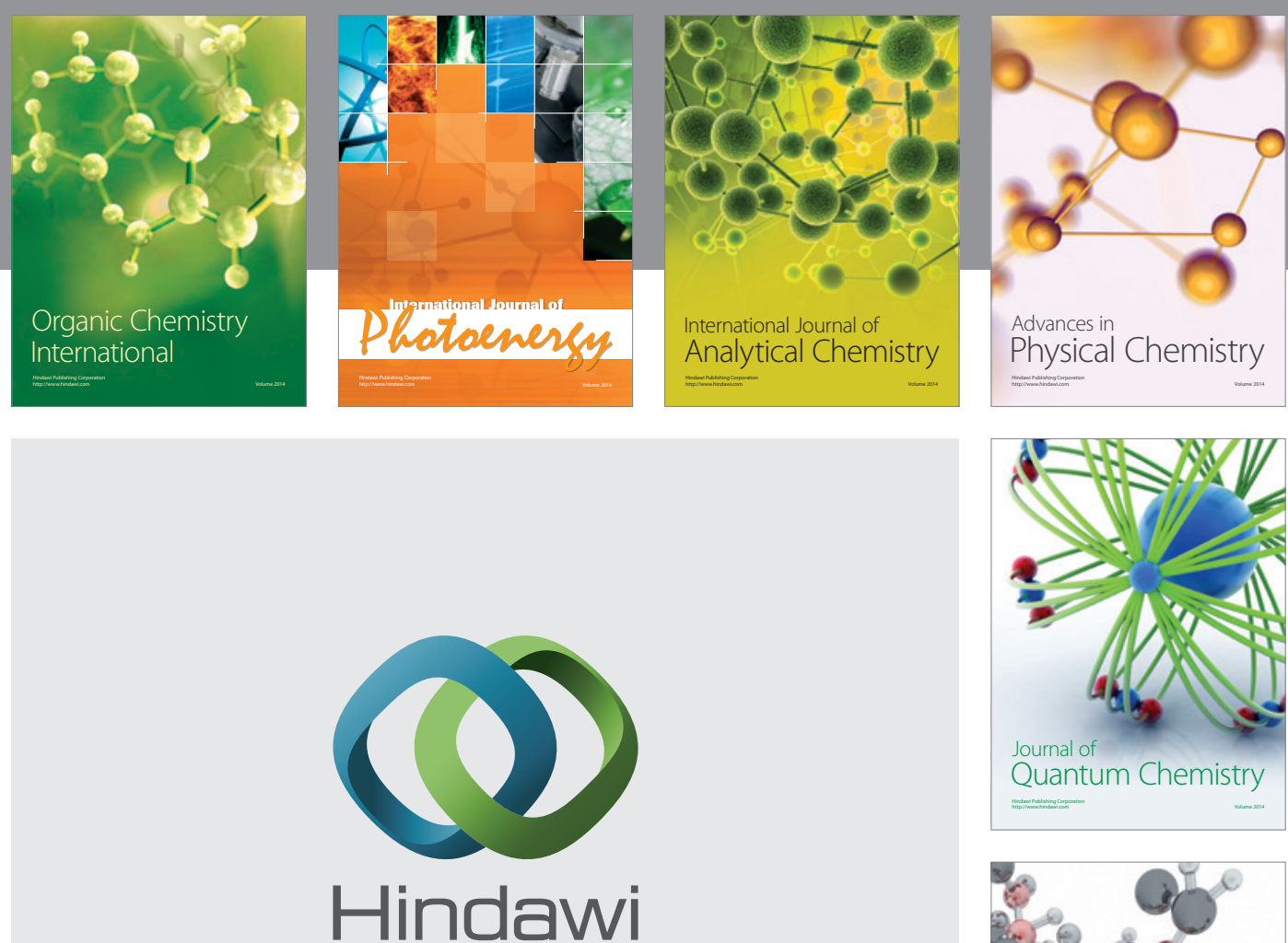

Submit your manuscripts at

http://www.hindawi.com

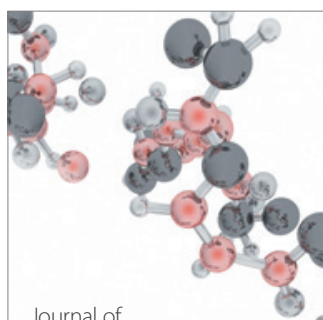

Analytical Methods

in Chemistry

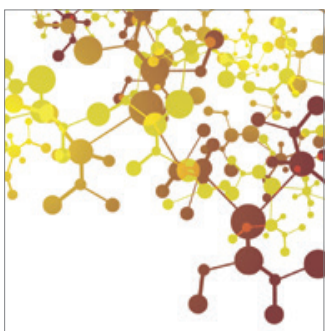

Journal of

Applied Chemistry

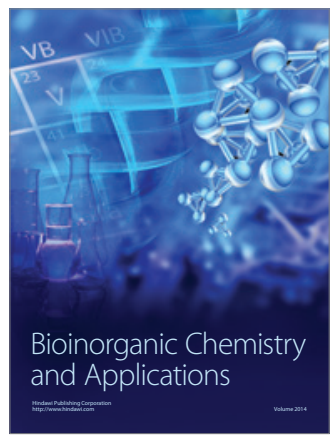

Inorganic Chemistry
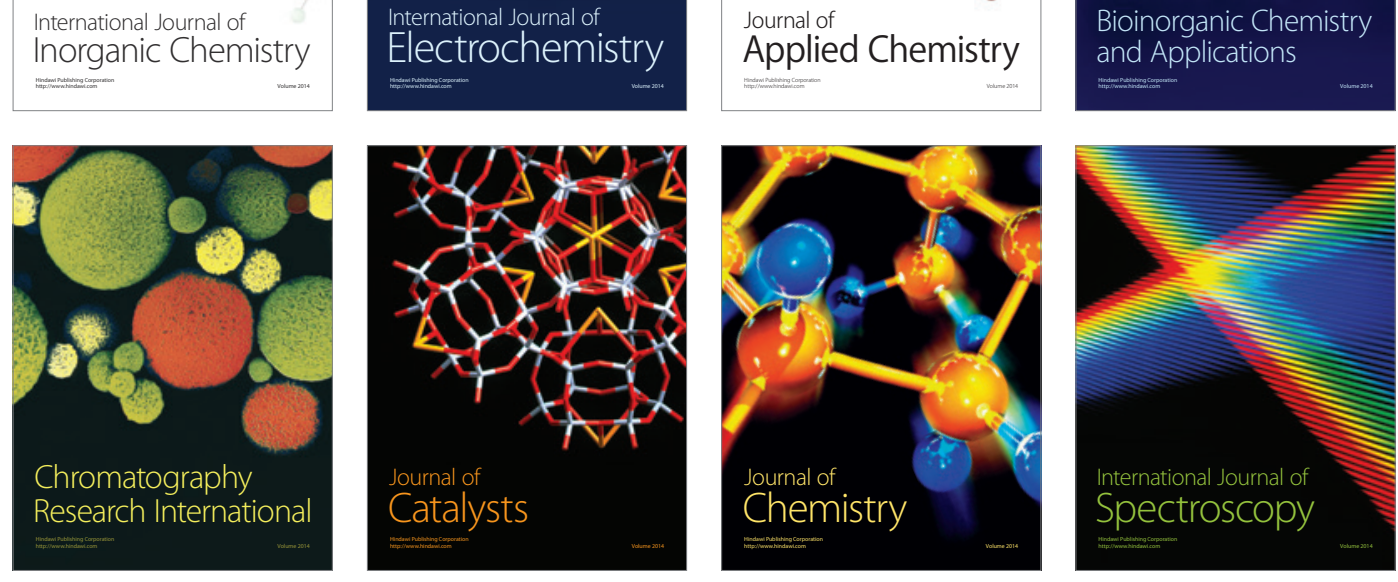